\title{
Innovación educativa y metodologías activas en educación secundaria: la perspectiva de los docentes de lengua castellana y literatura
}

\author{
Educational innovation and active methodologies in secondary \\ education: the perspective of teachers of Spanish language and \\ literature
}

\author{
Yolanda Abellán Toledo \\ Rosario Isabel Herrada Valverde \\ Universidad de Murcia
}

Recibido: 11/02/2016

Aceptado: 19/05/2016

\begin{abstract}
In the current educational context they are undertaking actions to improve teaching through new ways of acquiring knowledge and active involvement by students. The aim is to prepare ditizens to actively participate in society and to be able to respond to different situations that they might encounter in the future. Innovations in the educational context could enhance these actions, especially if teachers, students, families, and Educational Administration were involved in the process. In this paper we analyze the perceptions, ideas and personal experiences of a group of secondary school teachers assigned to the Department of Spanish Language and Literature in order to determine the aspects that could facilitate or hinder the educational innovation and theim plementation of teaching-learning active methodologies. Among the issues discussed are collaboration among teachers, changes in the subject of Spanish Language and Literature, or the relationship among teachers, students and families. From the results obtained, it is conduded that the teacher is a key player in the success of educational innovations implemented in secondary schools.
\end{abstract}

KEY WORDS: educational innovation; active methodologies; Spanish language and literature; secondary education; teachers.

\section{RESUMEN}

En el contexto educativo actual se están llevando a cabo acciones encaminadas a mejorar la enseñanza a través de nuevas formas de adquisición del conocimiento y de la implicación activa por parte del alumnado. Con ello se trata de preparar a la cudadanía para que participe activamente en la sociedad y sea capaz de responder a las distintas circunstancias que se le planteen en el futuro. Las innovaciones en el contexto educativo podrían favorecer dicha mejora, sobre todo si se implicaran en ello todos los agentes involucrados: el profesorado, el alumnado, las familias, y la Administración Educativa. En el presente artículo se analizan las percepciones, ideas y vivencias personales de un grupo de docentes de Edu cación Secundaria adscritos al Departamento de Lengua Castellana y Literatura acerca de algunos aspectos que podrían facilitar o dificultar la innovación educativa y la implem entación de metodologías activas de enseñanza-aprendizaje. Entre los aspectos que se analizan encontramos la colaboración entre el profesorado, los cambios en la materia de Lengua Castellana y Literatura, o la relación del profesorado con el alumnado y con las familias. A partir de resultados obtenidos, se conduye que el docente es un agente fundamental para el éxito de las innovaciones educativas que se emprendan en los centros educativos.

PALABRAS CLAVE: innovación educativa; metodologías activas; lengua castellana y literatura; educación secundaria; profesorado.

Dirección de correspondencia:

Yolanda Abellán Toledo, Universidad de Murcia. Dpto. Didáctica y Organización Escolar. Campus de Espinardo s/n, 30100 (Murcia). E-mail: yolanda.abellan@um.es

Rosario Isabel Herrada Valverde, Universidad de Murcia. Dpto. Didáctica y Organización Escolar. Campus de Espinardo s/n, 30100 (Murcia). E-mail: rherrada@um.es 


\section{Introducción}

La Lengua Castellana y Literatura constituye una de las materias más importantes del currículo de Educación Secundaria Obligatoria. Así, mientras que en la etapa de Educación Primaria se promueve que los alumnos adquieran habilidades lingüísticas tales como leer, escribir o hablar, en la etapa de Educación Secundaria se favorece que los alumnos afiancen sus competencias de comunicación lingüística, tanto oral como escrita (López y Martínez, 2012).

Diferentes estudios, como el informe PISA (MECD, 2013) han concluido que muchos de los estudiantes que finalizan su etapa de Educación Secundaria carecen de las competencias de comprensión lectora. Resulta evidente la necesidad de implementar medidas específicas que aborden dicha problemática, siendo fundamental que los agentes educativos se impliquen en ello. A esto se une las altas tasas de abandono escolar (Casquero y Navarro, 2010), que hacen que el docente deba promover acciones encaminadas a favorecer el "enganche" del alumnado (González, 2010) en su proceso de enseñanza-aprendizaje, y en las diferentes materias que componen el currículo. Para ello, dichos docentes podrían adoptar diversas medidas innovadoras en el aula. En este sentido, cabe indicar que gran parte de las investigaciones relativas a la innovación en el aula se han focalizado en el análisis de los rasgos personales del profesorado que podrían favorecer la innovación (Monge, Montalvo, y Gómez, 2015). Además de esos rasgos personales, algunas investigaciones destacan que el discurso sexista/ideológico del profesorado de Lengua Castellana y Literatura puede influir en sus relaciones con los alumnos (López, Madrid, y Encabo, 1998).

El presente trabajo analiza la visión general de un conjunto de docentes del Departamento de Lengua Castellana y Literatura de dos IES públicos situados en la Región de Murcia sobre el desarrollo de la materia y de cara a implementar acciones innovadoras en el aula, a la vez que se analiza la predisposición/uso de metodologías activas de enseñanza-aprendizaje (aprendizaje cooperativo, etc

\section{Innovación Educativa}

La innovación educativa surge en el contexto de la investigación y se desarrolla como "un elemento de creación de nuevos conocimientos, productos y procesos" (Gros y Lara, 2009, p. 225), de ahí que adquiera múltiples significados según dónde se sitúe. En este sentido, González y Escudero (1987) señalan que la innovación es algo más que un cambio superficial y que pretende alterar ideas, concepciones y prácticas, entre otras cuestiones, con el fin de renovar el sistema educativo. Por su parte, Gros y Lara (2009) opinan que:

La innovación no puede ser una práctica aislada. Un proyecto de innovación responde a una necesidad de diferenciación estratégica. La diferenciación es la meta y la innovación es el proceso para conseguirla. Por consiguiente, para que una organización sea innovadora ha de sistematizar y ejecutar de forma consciente y controlada su desarrollo estratégico. Los términos riesgo, confianza, colaboración, diferenciación, sostenibilidad, valor, calidad, son parte indisociable de la innovación. (p. 227)

Martínez Bonafé (2008) sitúa el punto de mira sobre el docente, como protagonista de la innovación:

El deseo que mueve a un docente a intentar mejorar su práctica profesional, más allá de una técnica o una teoría y siempre acompañado de una finalidad educativa [...] la innovación es un proceso histórico y político, problematizador, individual pero también cooperativo y con significados diversos e incluso a veces contradictorios entre sí. (p. 78)

Así pues, la innovación educativa implicaría la introducción de cambios para la mejora, bien relacionados con el sistema educativo en general, y con la práctica docente en particular. Además, existen ciertos factores a considerar que podrían favorecer o dificultar el éxito de las innovaciones 
(Carbonell, 2001). Entre los factores que promueven el éxito se encuentran los siguientes: equipos docentes fuertes y estables; comunidad educativa dispuesta al cambio; ambiente de bienestar; y creación de oportunidades y posibilidades para que las innovaciones se vivan con intensidad. En cambio, algunos factores que dificultan las innovaciones son: el pesimismo y malestar docente; las rutinas del profesorado; los efectos perversos de las reformas; y las paradojas del doble currículo.

Por otra parte, para delimitar el concepto de innovación, es preciso también diferenciarlo de otros conceptos que suelen ser utilizados como sinónimos, generando confusión. Para ello, clarificaremos modernización y mejora en relación con el concepto de imnovación.

En lo que respecta al concepto de modernización, en el ámbito escolar se relaciona con la instalación de ordenadores, realización de talleres, etc. De esta forma, una innovación en el aula puede conllevar la modernización de la misma, pero una modernización no tiene por qué implicar una innovación. De hecho, según Carbonell (2001), una modernización de la escuela (incluir ordenadores en las aulas, cultivar un huerto, etc.) no tiene por qué modificar las concepciones sobre enseñanza-aprendizaje, y podría derivar, en última instancia, en ofertas de marketing o falsas innovaciones para atraer al alumnado y estar a la moda. El verdadero cambio, según este autor, sería conseguir que el profesorado pusiera en entredicho sus pensamientos, actitudes y hábitos.

Algunos autores advierten de cierta confusión entre el concepto de modernización y el de innovación, y centrándose en el ámbito de las nuevas tecnologías, consideran que su uso no tiene por qué conllevar a una innovación: "no podemos pensar que esta [innovación] se produce solo a partir de la incorporación de la tecnología, concepto este muy arraigado en el ámbito educativo que ha conducido a enormes errores de apreciación y desarrollo" (Gros y Lara, 2009, p. 235).

Por otra parte, refiriéndonos específicamente al concepto de mejora, y teniendo en cuenta que se considera que el centro educativo debe ser el centro de los procesos de cambio, la mejora de la escuela supone, según Murillo (2002):

(...) el incremento de los resultados de los alumnos y los docentes [...] Para los alumnos, los resultados deberían ser: pensamiento crítico, capacidad para aprender, autoestima, etc., tanto como mejora en los exámenes o en los resultados de pruebas externas. Para los docentes podrían ser el incremento del trabajo en equipo, las oportunidades para el aprendizaje profesional y un aumento de la responsabilidad. (p. 17)

En España, según este autor, ha habido poco movimiento para la mejora educativa, sobre todo debido a la escasa autonomía de los centros a la hora de tomar decisiones, a la ausencia de políticas de apoyo y a la falta de compromiso interno. No obstante, hemos pasado de un sistema educativo en el que el centro apenas tenía poder de decisión, a otro donde el centro dispone de cierta autonomía en determinados campos, como, por ejemplo, en la iniciación de un proceso de cambio a partir de la reflexión sobre el currículo, el cambio en la organización o en cuanto a la formación del profesorado.

Por tanto, volviendo a la delimitación entre conceptos, una mejora se puede "planificar y es posible predecir los resultados de una forma bastante precisa. En cambio, innovar se trata no solo de mejorar un proceso o un producto sino que implica generar un verdadero cambio. [...] implica asumir riesgos" (Gros y Lara, 2009, p. 225).

Es importante considerar a los docentes para llevar a cabo acciones innovadoras, pues si parten de este colectivo tendrán más probabilidades de tener éxito. De hecho, cuando la innovación parte únicamente de la iniciativa de la Administración Educativa corre el riesgo de quedar relegada a "tomar las medidas necesarias de política educativa y dotar a la escuela pública de los recursos suficientes para que el profesorado pueda llevar a cabo las innovaciones bajo las necesarias condiciones de calidad" (Carbonell, 2001, p. 12). Una innovación educativa debe llevarse a la práctica y, por lo tanto, los promotores más directos para su éxito van a ser los docentes, que precisan contar con el apoyo de la Administración Educativa y de otros organismos para que propicien dicha innovación de forma indirecta. 
Por otro lado, es necesario favorecer el cambio de perspectiva del profesorado, que lleve a la transformación de sus actitudes, de la organización en el sistema educativo y del trabajo de los docentes en las aulas. Este cambio es determinante, más teniendo en cuenta que tanto la Ley Orgánica de Educación (LOE, 2006) como la Ley Orgánica para la Mejora de la Calidad Educativa (LOMCE, 2013) se plantean la educación por competencias, y por tanto es preciso que los educadores se centren en la comprensión y el manejo de los procesos de cambio educativo, mejo ra e innovación escolar.

También hay que tener presente que desde los propios centros educativos deben favorecerse procesos de reflexión, coordinación, participación y consenso que propicien la puesta en marcha y desarrollo de innovaciones educativas, en las que se implicaría el profesorado y otros agentes educativos. Michavila (2009) opina que tanto los profesores como los centros en su conjunto son decisivos para realizar las innovaciones educativas pertinentes y necesarias. Así, un trabajo coordinado del profesorado, incluyendo la participación de la comunidad educativa y del centro en su conjunto, hará que los alumnos puedan disfrutar de un proceso de enseñanza-aprendizaje enriquecedor, motivador y significativo. Ciertamente, una mayor colaboración entre docentes posibilitará mayores oportunidades para que la innovación desarrollada sea exitosa, si bien no podemos obviar que existen diferentes tipos de colaboración entre el profesorado, así como de trabajo colaborativo, como por ejemplo la denominada docencia compartida (Durán y Giné, 2011).

Por otro lado, el uso de nuevos recursos didácticos también potencia una docencia más innovadora, pero siempre que las Tecnologías de la Información y de la Comunicación (TIC) se utilicen para que el alumno amplíe, exprese y comunique a otros lo aprendido. Para ello, es fundamental la integración de las TIC junto con los demás elementos curriculares, y en particular, incorporar las TIC en la metodología docente de manera que se traduzcan realmente en experiencias innovadoras y no en simples herramientas expositivas o transmisoras de la información. Adell (2004) señala tres metáforas básicas: Internet como biblioteca; Internet como imprenta; Internet como canal de comunicación y plataforma de relación. Por consiguiente el docente debe conocer estas funciones para ofrecer oportunidades para el desarrollo de la competencia digital entre el alumnado, establecida tanto en la LOE (2006) como en la LOMCE (2013).

No obstante, autores como Fuentes, Ortega y Lorenzo (2005) afirman que existe cierta tecnofobia entre los docentes a la hora de aplicar las nuevas tecnologías en sus clases y que estos suelen tener escasa formación inicial en medios y tecnologías.

\section{Metodologías Activas}

Entre los diversos aspectos que podrían favorecer el cambio o mejora en el sistema educativo, podemos destacar el papel de las metodologías activas. Tradicionalmente se han promovido metodologías de enseñanza en las que el profesor es emisor y transmisor de los conocimientos, mientras que el alumno es quien recibe y asimila dichos conocimientos, actuando de forma pasiva en su proceso de aprendizaje. En cambio, cuando se implementan metodologías activas, el alumno se convierte en el protagonista, involucrándose e interviniendo en su proceso de aprendizaje, mientras que el docente actuaría como mediador, tutor, apoyo, guía, etc. Según Fernández March (2006, p. 39) "el perfil apropiado del estudiante viene caracterizado por los siguientes elementos: aprendiz activo, autónomo, estratégico, reflexivo, cooperativo, responsable". De hecho, el aprendizaje activo otorga mayor importancia a la aplicación de conocimientos en contextos significativos, lo que estaría en consonancia con los actuales planteamientos de las competencias-clave, también recogidas en la actual ley de educación.

Entre las metodologías activas más utilizadas y conocidas se encuentra el aprendizaje cooperativo, que se caracteriza por el uso de grupos reducidos de alumnos que trabajan de forma unificada para mejorar su propio aprendizaje y el de los demás. Los docentes evalúan el trabajo cooperativo bajo determinados criterios, que según Johnson, Johnson y Holubec (1999) deberían especificar los objetivos de la clase, tomar una serie de decisiones previas a la enseñanza, explicar a 
los alumnos la tarea y la interdependencia positiva, supervisar el aprendizaje, entre otros aspectos. Estos autores también afirman que, al cooperar, el rendimiento de los alumnos es más elevado que cuando se utilizan métodos competitivos o individualistas, ya que el alumnado desarrolla un nivel de razonamiento superior, pensamiento crítico y mayor motivación intrínseca. Pujolás (2004) señala algunas ventajas del aprendizaje cooperativo, entre ellas, el desarrollo de relaciones interpersonales; la aceptación de diferencias; la toma de perspectiva social; el desarrollo de la creatividad y la autoestima

\section{Innovación educativa y metodologías activas en la materia de lengua castellana y literatura en Educación Secundaria}

Centrándonos en el área de Lengua Castellana y Literatura, Núñez (2002) apunta que el objetivo principal en la educación lingǘstica de la enseñanza obligatoria es mejorar los usos discursivos de los estudiantes a través de una serie de destrezas que las personas suelen utilizar de manera usual en la vida cotidiana para comunicarse. Por consiguiente, si se le está pidiendo al alumnado que se adapte a un perfil comunicativo, es lógico que también cambie el papel del profesorado. Esto implica redefinir algunos aspectos tales como su propia función, la responsabilidad sobre el contenido, la relación alumno-profesor, la metodología, incluso la planificación, siendo necesario el trabajo colaborativo entre docentes para que tomen decisiones conjuntas respecto a las estrategias metodológicas y criterios de evaluación.

Así pues, se necesita un cambio, una innovación metodológica en el área de Lengua Castellana y Literatura en Educación Secundaria, pues los contenidos suelen ser los mismos, y el cambio se ha centrado bastante en el formato (Carbonell, 2001). En concreto, el uso de las TIC ha favorecido cambios a la hora de plantear las tareas, por ejemplo, permitiendo que alumnos hagan uso de plataformas virtuales para descargar actividades, subir las soluciones, etc.

En la rama de la materia centrada en la Literatura, se puede apreciar que se centra más en la adquisición de unos datos memorísticos en el ámbito educativo. En contraposición, Margallo (2012) considera fundamental al "lector literario", que implica favorecer hábitos lectores para comprender los textos literarios de forma significativa. Desde esta perspectiva, esta autora (Margallo, 2012, p. 140) afirma que "este desplazamiento exige a su vez una renovación metodológica, puesto que las actividades centradas en la reproducción memorística y en la aplicación de técnicas de comentario de texto no parecen las más adecuadas para la formación de lectores literarios".

Por otra parte, hay que recordar el auge de las TIC, gracias a las cuales se están llevando a cabo numerosos proyectos de innovación en los centros educativos. Un ejemplo es "Caza del Tesoro. Aprendo a navegar...por el Club de Lectores Kirico", de Zayas y Pérez Esteve (2010). Además, hay que subrayar que las TIC han ayudado a crear nuevas actividades que son de interés para el alumnado, propiciando que lean y/o que escriban.

En cuanto a la rama de la materia centrada en Lengua, el foco de atención de la didáctica lingüística suele estar en la forma de enseñar la gramática, que tradicionalmente se ha abordado desde una perspectiva centrada en la descripción de categorías gramaticales y en el análisis sintáctico, empleando actividades repetitivas y poco motivadoras para el alumno. Con todo, actualmente se pretende elegir un camino que lleve hacia la competencia lingüística y comunicativa de los estudiantes. Como señala Zayas (2012, p. 76), "el objetivo no es estudiar gramática, sino reflexionar sobre el modo de usar la lengua para hacer determinadas cosas, como componer el prólogo de una antología de poemas, un programa de mano para un recital poético, una crítica teatral, etc".

Cabe destacar que algunas investigaciones han analizado la implementación de metodologías activas en este contexto. Así, Sierra (2012) propone una serie de actividades en las que el alum nado de segundo curso de enseñanza secundaria obligatoria pongan en marcha una radio online con contenidos curriculares de la asignatura Lengua Castellana y Literatura, haciendo uso de un podcast, cuyos contenidos serán accesibles a través de un blog. El objetivo de esta propuesta es que los 
estudiantes trabajen de forma cooperativa en grupos heterogéneos, asumiendo diferentes roles y responsabilidades individuales, para llegar a alcanzar objetivos comunes. En otra investigación, Caro (2014) señala que la educación literaria es una ciencia social humanista que favorece un aprendizaje cooperativo y creativo. Dicha autora propone un modelo didáctico orientado a la adquisición de competencias en el que se combina el aprendizaje basado en proyectos de innovación con tareas de lectura significativa. Dicho modelo es validado a través del desarrollo de varias experiencias en Educación Primaria y Secundaria.

\section{Marco metodológico}

En el presente estudio se pretende conocer cómo se implementan las innovaciones educativas y las metodologías activas de los docentes de Lengua Castellana y Literatura de los Institutos de Educación Secundaria (IES) de la Región de Murcia. De manera específica, los objetivos de esta investigación han sido los siguientes:

- Identificar qué saben los profesores de Lengua Castellana y Literatura de los IES acerca de la innovación educativa.

- Identificar qué saben estos profesores sobre metodologías activas.

- Analizar los cambios que han producido o deberían producirse en la materia de Lengua Castellana y Literatura en los últimos años en lo que respecta a innovación educativa y metodologías activas, desde la experiencia de sus protagonistas.

- Determinar la influencia de ciertos factores para el éxito o no de la innovación educativa y/o de metodologías activas, según los participantes en esta investigación.

Este estudio se sitúa dentro del enfoque cualitativo, y en particular en el paradigma fenomenológico o etnográfico puesto que se pretende "comprender lo que ocurre en diferentes contextos humanos en función de lo que las personas interpretan sobre ellos y los significados que otorgan a lo que les sucede" (Martínez González, 2007, p. 31). Además, se ha optado por un estudio de casos, bastante propio de las Ciencias Sociales, que se refiere al estudio de un caso singular para llegar a comprender su actividad en circunstancias concretas (Stake, 1999). Además se utiliza este método porque se pretende comprender y entender lo que ocurre en el ámbito educativo, en este caso, para poder mejorar los procesos educativos (Martínez González, 2007).

Como instrumento de recogida de información se optó por una entrevista semi-estructurada, para la que se creó una guía que fue sometida a juicio de expertos. Entre los temas que se preguntaban se encuentran la relación actual del docente con el alumnado, las familias y la Administración Educativa; los cambios producidos en la materia de Lengua Castellana y Literatura; la innovación y la colaboración docente; y las metodologías tradicionales en contraposición con las activas.

En cuanto al contexto y a los participantes, en este estudio de casos se ha contado con dos IES públicos de la Región de Murcia. El primero está situado en una población rural que centra su actividad económica mayormente en las labores agrícolas. De este centro han participado todos los tres docentes (dos mujeres y un hombre) del Departamento de Lengua Castellana y Literatura.

El segundo centro se encuentra en el casco urbano de una ciudad industrial. Respecto a los participantes, también se ha contado con los cuatro docentes (tres mujeres y un hombre) que componen el Departamento de Lengua Castellana y Literatura de este Instituto.

Cabe señalar que todos los docentes han participado de forma voluntaria en esta investigación, y que se realizaron entrevistas individuales, que fueron grabadas en audio con el consentimiento de los informantes.

Tras la recogida de información se optó por el análisis de contenido, en el que se realizó un análisis textual basado en la traducción de los discursos, utilizando dos procedimientos: la descripción y la trascripción. En este análisis textual se pretende recuperar todos los matices del discurso, de ahí que se base en el análisis de contenido como instrumento que recoge información 
mediante el método científico, pues como señala Abela (2002, p. 2) "debe ser, sistemática, objetiva, replicable y válida". Con todo, lo que pretende el análisis de contenido es analizar lo observado y lo producido, además de la interpretación o análisis de los datos.

Seguidamente se ha clasificado la información obtenida en categorías, creando grupos temáticos con estos datos siguiendo un criterio de analogía. Hay que señalar que las categorías de análisis definidas en un principio experimentaron ciertos cambios a medida que se desarrolló el proceso de análisis de la información. El sistema definitivo de categorías, y dentro de ellas, de subcategorías, es el que ha estructurado los resultados y el análisis de datos, que son presentadas en el siguiente apartado.

Con objeto de mantener el anonimato de los participantes y ceñirnos a los objetivos planteados, y puesto que no se pretende hacer un estudio comparativo entre centros, cuando se hagan referencia a estos informantes se identificaran como Entrevistado N, por ejemplo, Entrevistado 1, Entrevistado 2, etc.

\section{Resultados}

En este apartado, se explicarán y analizarán los resultados obtenidos a partir de las categorías temáticas resultantes de este estudio, y que son las siguientes: alumnado, familias y Administración educativa; cambios en la materia de Lengua Castellana y Literatura; innovación y colaboración docente; y metodologias activas frente a metodologías tradicionales.

La primera categoría es la relación que mantiene el docente con el alumnado, las familias y la Administración Educativa. Respecto a la relación entre el profesor y el alumnado, según nuestros informantes, se ha deteriorado bastante por la falta de respeto y de disciplina del alumnado. El Entrevistado 1 señala: "ahora los alumnos son más disruptivos"; el Entrevistado 3 afirma que existen "muchos problemas de indisciplina y los alumnos cada vez saben menos". Esto puede deberse a los constantes cambios sociales que suelen dejar de lado los principios y los valores, o a que los alumnos no encuentren conexión entre lo que aprenden en el centro educativo y lo que viven en su día a día (Torrego, 2012). El Entrevistado 7 afirma que: "hay una serie de problemas sociales que dificultan el proceso de enseñanza y aprendizaje para los alumnos".

En cuanto a la relación entre las familias y el profesorado, existe cierta dejadez y escasa implicación por parte de las familias, y cuando hay implicación suele ser para reprochar algo al profesor. Tal y como afirma el Entrevistado 5: "las familias de ahora parece que están deseando pillarnos en alguna falta (...) muchas veces incluso nos sentimos inseguros". Estos docentes demandan una mayor colaboración de las familias en los centros, pues este sería uno de los factores clave para el éxito de esa innovación educativa de la que hablábamos con anterioridad. Los docentes también declaran estar cansados de los constantes cambios en la legislación educativa, porque consideran que perjudican al alumno, sobre todo por los recortes que se han producido en educación, lo que ha causado que se hayan suprimido muchos apoyos y la ratio profesor-alumnos haya aumentado, entre otros aspectos. Además, según afirma el Entrevistado 7, "en la legislación se dice de una cosa y luego en la práctica se hace otra".

Por consiguiente, en este malestar podrían estar influyendo los cambios legislativos que se quieren llevar a cabo en el sistema educativo y que difícilmente serán asumidos por todos los docentes, tal vez porque no los consideran imprescindibles o por no disponer de las condiciones laborales adecuadas para llevarlos a cabo. Esto conlleva que no exista una base sólida sobre la que comenzar a innovar.

La segunda categoría está relacionada con los cambios en la materia de Lengua Castellana y Literatura. Según nuestros informantes, los contenidos siguen siendo los mismos que hace diez años. El Entrevistado 1 echa en falta que el alumno trabaje más con los textos y no con la historia de la literatura siguiendo un modelo de "lector literario", en el sentido propuesto por Margallo (2012). Por otro lado, algunas opiniones se centran en los cambios que se han producido en los últimos diez años en los criterios de evaluación. Por ejemplo, el Entrevistado 6 y el Entrevistado 7, que se decantan por aplicar metodologías más innovadoras en las aulas, son también más críticos y 
consideran importante evaluar el proceso de enseñanza-aprendizaje y tener en cuenta el desarrollo integral del alumno, por lo que afirman que sí que han cambiado estos criterios a lo largo del tiempo. En cambio, los docentes que aplican metodologías más tradicionales en las aulas, tales como el Entrevistado 3 y el Entrevistado 5, afirman seguir evaluando igual que lo solían hacer, centrándose sobre todo en el resultado final, y afirman que no se han producido cambios en este sentido. Hay que destacar que el principal cambio que los docentes observan en la materia de Lengua Castellana y Literatura es la introducción de las nuevas tecnologías, como por ejemplo, la pizarra digital o el uso de ordenadores en el aula. Como afirma el Entrevistad o 5, "algunos aspectos han evolucionado, sobre todo por las nuevas tecnologías". Por tanto, los cambios más destacados han sido en cuanto a medios didácticos, haciéndose notar el uso de las TIC, que han pasado de ser solo recomendables a ser imprescindibles (Delgado, Gomis y Sánchez, 2013).

La tercera categoría hace referencia a la Innovación y la Colaboración docente, en la que se puede destacar que la mayoría de informantes confunden el término de innovación con el uso de las nuevas tecnologías, es decir, con el concepto de modernización, en el sentido al que aludía Carbonell (2001). Tal es el caso del Entrevistado 2 que alude: "yo he vivido la pizarra de tiza, luego he pasado a las pizarras de vileda y ahora tenemos las pizarras digitales. Eso podría considerarse una innovación". Algunos docentes parecen no tener claro cómo integrar las TIC en el aula para que se conviertan en una experiencia innovadora. De hecho, más bien utilizan los medios tecnológicos como otra forma de exponer los contenidos, tal es el caso del Entrevistado 3: "ya no hacemos fotocopias, sacamos los textos en las pizarras digitales". En este sentido se podría decir que los docentes no utilizan las TIC de forma innovadora (Fuentes, Ortega y Lorenzo, 2005).

Respecto a la colaboración docente y la colaboración entre Departamentos, se suele centrar en actividades extraescolares, dejando de lado otro tipo de colaboración, por ejemplo en cuanto a programaciones didácticas. Algunos docentes conocen y en ocasiones se han implicado en otro tipo de colaboración, como la que se deriva de los proyectos de innovación, en los que el papel del equipo docente en su conjunto son decisivos para el éxito del proyecto (Escudero, 1988). El Entrevistado 6 menciona: "aquí tenemos proyectos en común. Este año yo no colaboro, pero el año pasado sí estuvimos entre Historia y Lengua. Mi compañera creo que sí colabora este año, y tienen un proyecto que va realizando un grupo de trabajo durante todo el curso, con ayuda del Centro de Profesores y Recursos".

Por otra parte, los docentes señalan que no suelen participar en cursos de formación del profesorado por diversas razones, entre las que se encuentra el no tener tiempo suficiente para realizarlos, lo que conlleva que no se actualicen en este sentido. El Entrevistado 7 indica que el profesorado no tiene la formación necesaria para abordar los problemas actuales: "el profesorado no ha recibido una formación didáctica específica, sino que directamente después de obtener su título o su licenciatura, se ha puesto a dar clase y lo ha hecho como buenamente ha podido y ha aprendido a lo largo de los años". Los profesores que no se actualizan tampoco pueden comprender o llevar a cabo los cambios necesarios en el sistema educativo, puesto que los alum nos y la sociedad en su conjunto está en continuo cambio. En cualquier caso, el docente podría actualizarse, ya que tiene a su disposición los mencionados cursos de formación que ofrecen los CPR, o podría participar en proyectos de innovación docente.

Teniendo en cuenta lo expuesto hasta ahora, difícilmente podrá desarrollarse con éxito una innovación educativa, ni implementarse adecuadamente metodologías activas o desarrollar proyectos innovadores, pues, como afirma Núñez (2002) no es solo el alumnado el que debe adaptarse, sino que el profesorado revise el papel que desempeña, su responsabilidad sobre el contenido, e incluso la planificación que debe desarrollar para favorecer esa colaboración entre docentes.

Por último, vamos a referirnos al uso de metodologías tradicionales frente a metodologías activas. Algunos docentes opinan que siguen predominando las metodologías tradicionales por diversos motivos: porque el currículo no demanda otra clase de metodología (Entrevistado 1); por desconocer otra metodología que no sea la tradicional (Entrevistado 3); por la elevada ratio 
profesor-alumnado lo que hace que no se pueda utilizar otra metodología (Entrevistado 4); y por preferir aplicar únicamente metodologías tradicionales (Entrevistado 5).

En contraposición, algunos docentes combinan metodologías tradicionales e innovadoras según la situación y las necesidades (Entrevistado 2 y Entrevistado 6), mostrándose interesados en adecuar la enseñanza para que los alumnos aprendan utilizando estrategias diferentes, motivándoles a aprender y presentándoles la materia de forma más atractiva (Entrevistado 6 y Entrevistado 7). El Entrevistado 6 afirma que en sus clases le está dando muy buenos resultados trabajar en grupos, y que los alumnos se tutelen por parejas, entendiendo que el cambio está en hacer que aprendan entre ellos de manera activa. Esta es una técnica cooperativa que se apoya en el aprendizaje cooperativo, y que se denomina "tutoría entre iguales" (Durán y Vidal, 2004). El Entrevistado 7 aplica en clases el trabajo de los contenidos de forma deductiva y la realización de debates.

\section{Conclusiones e implicaciones}

Teniendo en cuenta el análisis de de datos y los objetivos propuestos en la investigación, podemos hacer referencia a algunas conclusiones e implicaciones que son expuestas a continuación. En primer lugar, los docentes de Educación Secundaria de Lengua Castellana y Literatura a los que se refiere este estudio apenas están implementando innovaciones educativas y metodologías activas en las aulas, y en ello parece tener cierto peso la escasa formación psicopedagógica del profesorado y la necesidad de una mayor colaboración entre el profesorado y la comunidad educativa en general. Hay que tener en cuenta que para propiciar la innovación educativa es fundamental el papel del docente, y es preciso que conozca y se forme en aspectos relacionados con metodologías innovadoras.

Sin embargo, del análisis de los datos se desprende que la mayoría de estos docentes confunden el concepto de modernización con el de innovación educativa, que algunos están interesados en los proyectos de innovación educativa y en las metodologías activas, pero que la mayoría no llega a implicarse del todo. Todos enfatizan la falta de interés de los estudiantes por la materia de Lengua Castellana y Literatura. Puede que una de las razones sea que el estudiante no encuentre conexión entre lo que estudia y su día a día fuera de las aula. Quizá el uso de metodologías activas, podrían mejorar la motivación y el interés del alumno en este sentido.

En segundo lugar, otra conclusión obtenida es que los cambios legislativos desorientan a estos docentes. Además, ciertos entrevistados afirman que no pueden llevarse luego a la práctica estas reformas por falta de apoyos, ratio elevada de alumnos, etc. Todo ello hace que exista cierto malestar en este sentido, y los cambios legislativos no parecen estar calando del todo en las prácticas desarrolladas por estos docentes.

En tercer lugar, todos los entrevistados coinciden en mostrar cierto interés por el uso de los nuevos medios didácticos en las aulas, y por una mayor colaboración entre el profesorado. No obstante, aún es necesario avanzar en este sentido, pues el interés por los nuevos medios didácticos se reduce al cambio de formato en la presentación de la información, mientras que a la colaboración entre Departamentos parece estar centrada en las actividades puntuales. En todo caso, la participación en proyectos de innovación parece estar favoreciendo una mayor implicación entre Departamentos.

Asimismo, en el área de Lengua Castellana y Literatura, la innovación entre los docentes sigue siendo escasa, a excepción de algunos docentes que, por ejemplo, están implementando técnicas vinculadas al aprendizaje cooperativo en sus aulas, mostrando así cierto interés hacia metodologías más participativas y activas. En este sentido se podría decir que se aprecia un cambio en algunos docentes, que está evolucionando hacia una enseñanza-aprendizaje adaptada a los tiempos actuales.

En definitiva, y para finalizar, cabe señalar que la labor del docente es clave para el éxito de las innovaciones educativas. Este es el tronco del que germinaría una mayor colaboración entre profesores, un mejor uso de metodologías activas, el conocimiento necesario para atender lo que demanda el alumno actual, la intervención de las familias en los centros y la realización de proyectos 
de innovación, todo ello contando con el apoyo de la Administración Educativa. Posiblemente la clave sea favorecer el cambio de mentalidad del docente para que pueda llevar a cabo su labor en colaboración, siendo su principal objetivo el alumno. Todo esto podría potenciarse en la práctica si el docente conoce, aplica y se implica en las innovaciones educativas, en proyectos educativos o en metodologías activas.

Por último, cabe mencionar que el estudio aquí presentado abre una interesante puerta en futuras investigaciones para comprender y entender lo que está sucediendo en los Institutos de Educación Secundaria, y en particular, en lo que respecta al área y profesorado de Lengua Castellana y Literatura, de cara a establecer mejoras en los procesos educativos.

\section{Referencias bibliográficas}

Abela, J.A. (2002). Las técnicas de análisis de contenido, una revisión actualizada. Fundación Centro de Estudios Andaluces. URL: http:// public.centrodeestudiosandaluces.es/pdfs/S200103.pdf

Adell, J. (2004). Internet en educación. Comunicación y Pedagogía. 200, 25-28. Recuperado de: http://elbonia.cent.uij.es/jordi/wpcontent/uploads/docs/Comunicacion_y Pedagogia def.pdf

Caro, M.T. (2014). La educación literaria de los clásicos y su proyección interdisciplinaria para el aprendizaje basado en competencias. Educatio Siglo XXI, 32(3), 31-50. Recuperado de: http:/ / revistas.um.es/educatio/article/download/210961/168161

Carbonell, J. (2001). La aventura de innovar. El cambio en la escuela. Madrid: Morata.

Casquero, A. \& Navarro, M.L. (2010). Determinantes del abandono escolar temprano en España. Un análisis por género. Revista de Educación, Número extraordinario, 191-213. Recuperado de: http://www.revistaeducacion.educacion.es/re2010/re2010 08.pdf

Delgado, B., Gomis, N. \& Sánchez, V. (2013). Percepción del uso de las Tecnologías de la Información y la Comunicación de los Maestros. En $\mathrm{M}^{\mathrm{a}} \mathrm{C}$. Cardona, E. Chiner y A.V. Giner (Eds.). Investigación e Innovación Educativa al Servicio de Instituciones y Comunidades Globales, Plurales y Diversas (pp. 863-870). AIDIPE: Universidad de Alicante.

Durán, D. \& Giné, C. (2011). La formación del profesorado para la educación inclusiva: Un proceso de desarrollo profesional y de mejora de los centros para atender la diversidad. Revista Latinoamericana de Inclusión Educativa, I(2), 153-170. Recuperado de: http://www.rinace.net/rlei/numeros/vol5-num2/Revista\%20Educacion\%20Inclusiva.pdf

Durán, D. \& Vidal, V. (2004). Tutoría entre iguales: de la teoría a la práctica. Barcelona: Graó.

Escudero, J. M. (1988). La innovación y la organización escolar. En R. Pascual (coord.) La gestión educativa ante la innovación y el cambio (pp. 84-99). Madrid: Narcea.

Fernández, A. (2006). Metodologías activas para la formación de competencias. Educatio siglo XXI, 24, 35-56. Recuperado de:

http://www.unizar.es/ice/images/stories/materiales/curso35 2009/Metodologiasactivas.pdf

Fuentes, J. A., Ortega, J. A. y Lorenzo, M. (2005). Tecnofobia como déficit formativo. Investigando la integración curricular de las TIC en centros públicos de ámbito rural y urbano. Educar, 36, 169-180. Recuperado de: www.raco.cat/index.php/Educar/article/download/39740/39578

González, Ma.T. (2010). El alumno ante la escuela y su propio aprendizaje: algunas líneas de investigación en torno al concepto de implicación. Revista Iberoamericana sobre Calidad, Eficacia y Cambio en Educación, 8(4), 10-31.

González, Ma T. \& Escudero, J. M. (1987). Innovación educativa: teorías y procesos de desarrollo. Barcelona: Humánitas.

Gros, B. \& Lara, P. (2009). Estrategias de innovación en la educación superior: el caso de la Universitat Oberta de Catalunya. Revista Iberoamericana de Educación. 49, 223-245. Recuperado de: http://www.rieoei.org/ rie49a09.pdf 
Johnson, D.W., Johnson, R.T. \& Holubec, E. J. (1999). El aprendizaje cooperativo en el aula. Argentina: Paidós.

Ley Orgánica 2/2006, de 3 de mayo, de Educación (BOE núm. 106, de 4 de mayo de 2006). Recuperado de: $\underline{\text { https:// www.boe.es/buscar/doc.php?id=BOE-A-2006-7899 }}$

Ley Orgánica 8/2013, de 9 de diciembre, para la Mejora de la Calidad Educativa (BOE núm. 295, de 10 de diciembre de 2013). URL: https:/ / www.boe.es/diario boe/txt.php?id=BOE-A-2013$\underline{12886}$

López, A. \& Martínez, A. (2012). Aprendizaje y Enseñanza de la Lengua Castellana y la Literatura: Currículo de Educación Secundaria Obligatoria. Contextos Educativos, 15, 27-40. Recuperado de: dialnet.unirioja.es/descarga/articulo/3972872.pdf

López, A., Madrid, J.M. \& Encabo, E. (1998). El discurso del profesorado del área de Lengua y Literatura ante la transmisión de géneros en la educación secundaria. Revista Fuentes, 1, 107-122. Recuperado de: https:// ojs.publius.us.es/ojs/index.php/fuentes/article/view/2294

Margallo, A.M. (2012). La educación literaria en los proyectos de trabajo. Revista Iberoamericana de Educación, 59, 139-156. Recuperado de: http:/ / www.rieoei.org/rie59a06.pdf

Martínez Bonafé, J. (2008). Pero ¿Qué es la innovación educativa? Cuadernos de Pedagogía, 375, 78-82. Recupardo de: http://imced.edu.mx/edocs/cp080113.pdf

Martínez González, R.A. (2007). La investigación en la práctica educativa: Guia metodológica de investigación para el diagnóstico y evaluación en los centros docentes. Madrid: CIDE - Ministerio de Educación y Ciencia.

MECD (2013). PISA 2012 Programa para la evaluación internacional de los alumnos. Informe Español. Volumen I: Resultados y Contexto. Recuperado de: http://www.mecd.gob.es/dctm/inee/internacional/pisa2012/pisa2012lineavolumeni.pdf?docu mentId $=0901 \mathrm{e} 72 \mathrm{~b} 81786310$

Michavila, F. (2009). La innovación educativa. Oportunidades y barreras. ARBOR Ciencia, $\begin{array}{lllll}\text { Pensamiento } y & \text { Cultura, } & \text { 185, } & \text { Recuperado de: }\end{array}$ http://arbor.revistas.csic.es/index.php/arbor/article/view/373/374

Monge, C., Montalvo, D. \& Gómez, P. (2015). Los conocimientos sobre rasgos de personalidad del profesorado como facilitadores de la innovación educativa: Estado del arte. Revista Fuentes, 16, 173-198. Recuperado de: http://institucional.us.es/fuentes/gestor/apartados revista/pdf/ numeros anteriores/zcxlemsj. $\mathrm{pdf}$

Murillo, F.J. (2002). La "Mejora de la Escuela": concepto y caracterización. En F. J. Murillo y M. Muñoz-Repiso (coords.) La mejora de la escuela: un cambio de mirada (pp. 15-51). Barcelona: Octaedro.

Núñez, M.P. (2002). Cuestiones teóricas y metodológicas sobre la selección y diseño de actividades para la educación lingüística. REsLA, Revista Española de Lingüística Aplicada, 15, 113-135. URL: http://servicios.educarm.es/templates/portal/ficheros/websDinamicas/154/activ.pdf

Pujolás, P. (2004). Aprender juntos alumnos diferentes. Los equipos de aprendiraje cooperativo en el aula. Barcelona: Octaedro.

Sierra, V. (2012). Una radio interactiva a través del aprendizaje cooperativo. Universidad de La Rioja. Servicio de Publicaciones.

Stake, R.E. (1999). Investigación con estudio de casos. Madrid: Morata.

Torrego, A. (2012). La utilización de los blogs como recurso educativo en el área de Lengua Castellana y Literatura. REIFOP-Revista Electrónica Interuniversitaria de Formación del Profesorado, 15 (4): 127-137. URL: http://www.redalyc.org/articulo.oa?id=217026243010

Zayas, F. (2012). Los géneros discursivos y la enseñanza de la composición escrita. Revista Iberoamericana de Educación, 59, 63-85. URL: http://www.rieoei.org/rie59a03.pdf

Zayas, F. y Pérez, P. (2010). Caza del tesoro. Aprendo a navegar... por el Club de Lectores Kiriko. Centro Virtual Leer.es. Ministerio de Educación, Cultura y Deporte. 


\section{Para citar este artículo}

Abellán Toledo, Y., \& Herrada Valverde, R.I. (2016). Innovación educativa y metodologías activas en educación secundaria: la perspectiva de los docentes de lengua castellana y literatura. Revista Fuentes, 18(1), 65-76. [Fecha de consulta: dd/mm/aa]. doi: doi: http://dx.doi.org/10.12795/revistafuentes.201 6.18.1.04 